\title{
The Analytical and Numerical Solutions of Differential Equations Describing of an Inclined Cable Subjected to External and Parametric Excitation Forces
}

\author{
Mohamed S. Abd Elkader ${ }^{1,2}$ \\ ${ }^{1}$ Department of Mathematics and Statistics, Faculty of Science, Taif University, El-Taif, \\ Kingdom of Saudi Arabia \\ ${ }^{2}$ Department of Engineering Mathematics, Faculty of Electronic Engineering, Menoufia University, \\ Menouf, Egypt \\ E-mail:moh_6_11@yahoo.com
}

Received October 21, 2011; revised November 22, 2011; accepted November 30, 2011

\begin{abstract}
The analytical and numerical solutions of the response of an inclined cable subjected to external and parametric excitation forces is studied. The method of perturbation technique are applied to obtained the periodic response equation near the simultaneous principal parametric resonance in the presence of 2:1 internal resonance of the system. All different resonance cases are extracted. The effects of different parameters and worst resonance case on the vibrating system are investigated. The stability of the system are studied by using frequency response equations and phase-plane method. Variation of the parameters $\alpha_{2}, \alpha_{3}, \beta_{2}, \gamma_{2}, \eta_{2}, \gamma_{3}, \eta_{3}$, $f_{2}$ leads to multi-valued amplitudes and hence to jump phenomena. The simulation results are achieved using MATLAB 7.6 programs.
\end{abstract}

Keywords: Perturbation Method, Resonance, Chaotic Response, Stability

\section{Introduction}

Cable structures play an important role in many engineering fields, such as civil, ocean and electric engineering. Arafat and Nayfeh [1] studied the motion of shallow suspended cables with primary resonance excitation. The method of multiple scales is applied to study nonlinear response of this suspended cables and its stability and the dynamic solutions. Some interesting work on the nonlinear dynamics of cables to the harmonic excitations can be found in the review articles by Rega [2,3]. Nielsen and Kierkegaard [4] investigated simplified models of inclined cables under super and combinatorial harmonic excitation and gave analytical and purely numerical results. Zheng, Ko and Ni [5] considered the super-harmonics and internal resonance of a suspended cable with almost commensurable natural frequencies. Zhang and Tang [6] investigated the chaotic dynamics and global bifurcations of the suspended inclined cable under combined parametric and external excitations. Nayfeh et al. [7] investigated the nonlinear nonplanar responses of suspended cables to external excitations. The equations of motion governing such systems contain quadratic and cubic nonlinearities, which may result in 2:1 and 1:1 internal resonances. Chen and $\mathrm{Xu}[8]$ investigated the global bifurcations of the inclined cable subjected to a harmonic excitation leading to primary resonances with the external damping by using averaging method. Kamel and Hamed [9], studied the nonlinear behavior of an inclined cable subjected to harmonic excitation near the simultaneous primary and 1:1 internal resonance using multiple scale method. Abe [10] investigated the accuracy of nonlinear vibration analyses of a suspended cable, which possesses quadratic and cubic nonlinearities, with 1:1 internal resonance. The nonlinear dynamics of suspend cable structures have been studied with 2:1 internal resonances by the authors [11,12]. Experimental studies of this problem have been conducted by Alaggio and Rega [13] and Rega and Allagio [14], however explicit stability regions for the semi-trivial solution have not been calculated analytically. Here, we use a modal model to compute the instability boundary for a range of excitation frequencies close to the 2:1 resonance for an inclined cable, including nonlinear modal interaction. The out-of-plane 
dynamic stability of inclined cables subjected to in-plane vertical support excitation is investigated by GonzalezBuelga et al. [15]. Perkins [16] examined the effect of one support motion on the three-dimensional nonlinear response. Using the Galerkin method, he constructed a two-degree-of-freedom model to analyze the 2:1 internal resonance. Lee and Perkins [17] extended the work to include second-order perturbations and multiple internal resonances. Still, the focus was on the 2:1 internal resonance, whereas the excitation was changed to a harmonically varying load per unit length acting in the static equilibrium plane. Lee and Perkins [18] also used a three-degree-of-freedom model to simulate non-linear response of suspended, inclined cables driven by planar excitation and determined the existence and stability of four classes of periodic solutions.

Eissa and Sayed [19-21] and Sayed [22], studied the effects of different active controllers on simple and spring pendulum at the primary resonance via negative velocity feedback or its square or cubic. Sayed and Hamed [23] studied the response of a two-degree-of-freedom system with quadratic coupling under parametric and harmonic excitations. The method of multiple scale perturbation technique is applied to solve the non-linear differential equations and obtain approximate solutions up to and including the second-order approximations. Sayed and Kamel $[24,25]$ investigated the effects of different controllers on the vibrating system and the saturation control to reduce vibrations due to rotor blade flapping motion. The stability of the obtained numerical solution is investigated using both phase plane methods and frequency response equations. Amer and Sayed [26], studied the response of one-degree-of freedom, non-linear system under multi-parametric and external excitation forces simulating the vibration of the cantilever beam. Variation of some parameters leads to multi-valued amplitudes and hence to jump phenomena. Sayed et al. [27], investigated the non-linear dynamics of a two-degree-of freedom vibration system including quadratic and cubic non-linearities subjected to external and parametric excitation forces. The stability of the system is investigated using both frequency response curves and phase-plane trajectories. The effects of different parameters of the system are studied numerically.

This work deals with model having two-degree-offreedom nonlinear system subjected to external and parametric excitation forces describes the vibrations of an inclined cable. The method of multiple scales perturbation is applied to obtain modulation response equations near the simultaneous principal parametric resonance in the presence of 2:1 internal resonance $\left(\Omega_{2} \cong 2 \omega_{2}\right.$ and $\left.\omega_{1} \cong 2 \omega_{2}\right)$. The stability of the proposed analytic nonlinear solution near the above case is studied and the stabil- ity condition is determined. The effect of different parameters on the steady state response of the vibrating system is studied and discussed from the frequency response curves. The numerical solution and chaotic responses of the nonlinear system of an inclined cable for some different parameters are also studied. A comparison with previously published work is included.

\section{Mathematical Analysis}

Our attention is focused on an elastic-sag hanging at fixed supports and excited by harmonic and parametric distributed vertical forcing in plane. The two-degree-offreedom describing the nonlinear dynamics of cable shown in Figure 1, can be written as:

$$
\begin{gathered}
\ddot{x}+2 c_{1} \dot{x}+\omega_{1}^{2} x+\alpha_{2} x^{2}+\beta_{2} y^{2}+\gamma_{2} x^{3}+\eta_{2} x y^{2}=0 \\
\ddot{y}+2 c_{2} \dot{y}+\omega_{2}^{2} y+\alpha_{3} x y+\gamma_{3} y^{3}+\eta_{3} x^{2} y \\
=f_{1} \cos \Omega_{1} t+y f_{2} \cos \Omega_{2} t
\end{gathered}
$$

where $x$ and $y$ denote in-plane and out-of-plane displacements, respectively, and dots denote derivatives with respect to the time $t$. The parameters $c_{1}$ and $c_{2}$ are the viscous damping coefficients, $\omega_{1}$ and $\omega_{2}$ are the natural frequencies associated with in-plane and out-ofplane modes $\Omega_{1}$ and $\Omega_{2}$ are the excitation frequencies, $f_{1}$ and $f_{2}$ are the excitation forces amplitude, $\alpha_{2}, \beta_{2}, \gamma_{2}$, $\eta_{2}, \alpha_{3}, \gamma_{3}$ and $\eta_{3}$ are the coefficients of nonlinear parameters. The linear viscous damping forces, the exciting forces and nonlinear parameters are assumed to be

$$
\begin{aligned}
& c_{1}=\varepsilon^{2} \hat{c}_{1}, c_{2}=\varepsilon^{2} \hat{c}_{2}, f_{n}=\varepsilon^{2} \hat{f}_{n}, \gamma_{s}=\varepsilon^{2} \hat{\gamma}_{s}, \hat{\eta}_{s}=\varepsilon^{2} \hat{\eta}_{s}, \\
& \hat{\alpha}_{s}=\varepsilon \hat{\alpha}_{s}, \beta_{2}=\varepsilon \hat{\beta}_{2} \\
& n=1,2 \quad s=2,3
\end{aligned}
$$

where $\varepsilon$ is a small perturbation parameter and $0<\varepsilon \ll 1$. For the convenience of the analysis of Equations (1)-(2), the non-dimensional parameter $\varepsilon$ is introduced. We can obtain

$$
\begin{gathered}
\ddot{x}+2 \varepsilon^{2} \hat{c}_{1} \dot{x}+\omega_{1}^{2} x+\varepsilon\left(\hat{\alpha}_{2} x^{2}+\hat{\beta}_{2} y^{2}\right) \\
+\varepsilon^{2}\left(\hat{\gamma}_{2} x^{3}+\hat{\eta}_{2} x y^{2}\right)=0 \\
\ddot{y}+2 \varepsilon^{2} \hat{c}_{2} \dot{y}+\omega_{2}^{2} y+\varepsilon \hat{\alpha}_{3} x y+\varepsilon^{2}\left(\hat{\gamma}_{3} y^{3}+\hat{\eta}_{3} x^{2} y\right) \\
=\varepsilon^{2}\left(\hat{f}_{1} \cos \Omega_{1} t+y \hat{f}_{2} \cos \Omega_{2} t\right)
\end{gathered}
$$

The parameters $\hat{\alpha}_{2}, \hat{\alpha}_{3}, \hat{\beta}_{2}$ are of the order of 1 and the parameters $\hat{c}_{1}, \hat{c}_{2}, \hat{\gamma}_{2}, \hat{\gamma}_{3}, \hat{\eta}_{2}, \hat{\eta}_{3}, \hat{f}_{1}, \hat{f}_{2}$ are of the order of 2. The approximate solution of Equations (3)-(4) can be obtained using the method of multiple scales [28]. Let

$$
\begin{aligned}
x(t ; \varepsilon)= & x_{0}\left(T_{0}, T_{1}, T_{2}\right)+\varepsilon x_{1}\left(T_{0}, T_{1}, T_{2}\right) \\
& +\varepsilon^{2} x_{2}\left(T_{0}, T_{1}, T_{2}\right)
\end{aligned}
$$



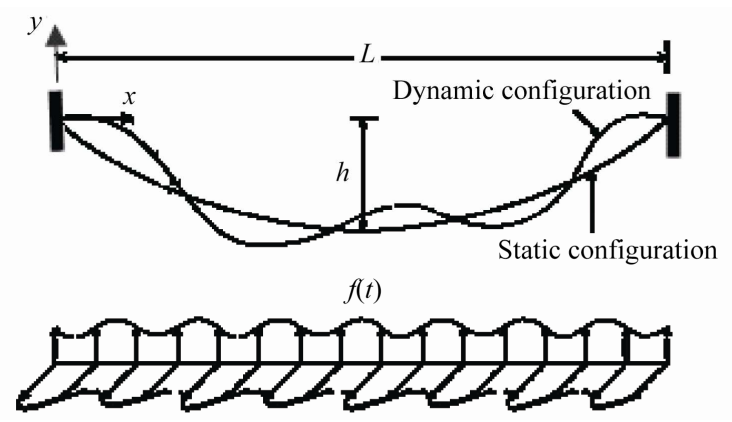

Figure 1. A schematic of inclined cable under combined excitations.

$$
\begin{aligned}
y(t ; \varepsilon)= & y_{0}\left(T_{0}, T_{1}, T_{2}\right)+\varepsilon y_{1}\left(T_{0}, T_{1}, T_{2}\right) \\
& +\varepsilon^{2} y_{2}\left(T_{0}, T_{1}, T_{2}\right)
\end{aligned}
$$

where, $T_{n}=\varepsilon^{n} t \quad(n=0,1,2)$ are the fast and slow time scales respectively. In terms of $T_{0}, T_{1}$ and $T_{2}$, the time derivatives transform according to

$$
\begin{aligned}
& \frac{\mathrm{d}}{\mathrm{d} t}=D_{0}+\varepsilon D_{1}+\varepsilon^{2} D_{2} \\
& \frac{\mathrm{d}^{2}}{\mathrm{~d} t^{2}}=D_{0}^{2}+2 \varepsilon D_{0} D_{1}+\varepsilon^{2}\left(D_{1}^{2}+2 D_{0} D_{2}\right)
\end{aligned}
$$

where $D_{n}=\partial / \partial T_{n}$. Substituting Equations (5)-(6) and (7) into Equations (3)-(4) and equating the coefficients of similar powers of $\varepsilon$ in both sides, we obtain the differential equations as follows:

Order $\left(\varepsilon^{0}\right)$ :

$$
\begin{aligned}
& \left(D_{0}^{2}+\omega_{1}^{2}\right) x_{0}=0 \\
& \left(D_{0}^{2}+\omega_{2}^{2}\right) y_{0}=0
\end{aligned}
$$

Order $\left(\varepsilon^{1}\right)$ :

$$
\begin{gathered}
\left(D_{0}^{2}+\omega_{1}^{2}\right) x_{1}=-2 D_{0} D_{1} x_{0}-\hat{\alpha}_{2} x_{0}^{2}-\hat{\beta}_{2} y_{0}^{2} \\
\left(D_{0}^{2}+\omega_{2}^{2}\right) y_{1}=-2 D_{0} D_{1} y_{0}-\hat{\alpha}_{3} x_{0} y_{0}
\end{gathered}
$$

Order $\left(\varepsilon^{2}\right)$ :

$$
\begin{aligned}
\left(D_{0}^{2}+\omega_{1}^{2}\right) x_{2}= & -D_{1}^{2} x_{0}-2 D_{0} D_{1} x_{1}-2 D_{0} D_{2} x_{0} \\
& -2 \hat{c}_{1} D_{0} x_{0}-2 \hat{\alpha}_{2} x_{0} x_{1}-2 \hat{\beta}_{2} y_{0} y_{1} \\
& -\hat{\gamma}_{2} x_{0}^{3}-\hat{\eta}_{2} x_{0} y_{0}^{2} \\
\left(D_{0}^{2}+\omega_{2}^{2}\right) y_{2}= & -D_{1}^{2} y_{0}-2 D_{0} D_{1} y_{1}-2 D_{0} D_{2} y_{0} \\
& -2 \hat{c}_{2} D_{0} y_{0}-\hat{\alpha}_{3}\left(x_{0} y_{1}+y_{0} x_{1}\right)-\hat{\gamma}_{3} y_{0}^{3} \\
& -\hat{\eta}_{3} y_{0} x_{0}^{2}+\hat{f}_{1} \cos \Omega_{1} T_{0}+y_{0} \hat{f}_{2} \cos \Omega_{2} T_{0}
\end{aligned}
$$

The solution of Equations (8)-(9) can be expressed in the complex form:

$$
\begin{aligned}
& x_{0}=A\left(T_{1}, T_{2}\right) \exp \left(i \omega_{1} T_{0}\right)+c c \\
& y_{0}=B\left(T_{1}, T_{2}\right) \exp \left(i \omega_{2} T_{0}\right)+c c
\end{aligned}
$$

where $c c$ denotes the complex conjugate of the preceding terms and $A, B$ are complex functions in $T_{1}$ and $T_{2}$ which determined through the elimination of secular and small-divisor terms from the first and second-order of approximations.

In this case, we analyze the case where $\Omega_{2} \cong 2 \omega_{2}$ and $\omega_{1} \cong 2 \omega_{2}$. To describe quantitatively the nearness of the resonances, we introduce the detuning parameters $\sigma_{1}$ and $\sigma_{2}$ according to $\Omega_{2}=2 \omega_{2}+\varepsilon \hat{\sigma}_{1}, \omega_{1}=2 \omega_{2}+\varepsilon \hat{\sigma}_{2}$. Substituting Equations (14)-(15) into Equations (10)-(11) and eliminating the secular terms leads to the solvability conditions for the first-order expansion as:

$$
\begin{array}{r}
2 i \omega_{1} D_{1} A+\hat{\beta}_{2} B^{2} \exp \left(-i \hat{\sigma}_{2} T_{1}\right)=0 \\
2 i \omega_{2} D_{1} B+\hat{\alpha}_{3} A \bar{B} \exp \left(i \hat{\sigma}_{2} T_{1}\right)=0
\end{array}
$$

After eliminating the secular terms, the particular solutions of Equations (10)-(11) are given by:

$$
\begin{array}{r}
x_{1}=\frac{\hat{\alpha}_{2}}{3 \omega_{1}^{2}} A^{2} \exp \left(2 i \omega_{1} T_{0}\right)-\frac{\hat{\alpha}_{2}}{\omega_{1}^{2}} A \bar{A}-\frac{\hat{\beta}_{2}}{\omega_{1}^{2}} B \bar{B}+c c \\
y_{1}=-\frac{\hat{\alpha}_{3}}{\left[\omega_{2}^{2}-\left(\omega_{1}+\omega_{2}\right)^{2}\right]} A B \exp \left(i\left(\omega_{1}+\omega_{2}\right) T_{0}\right)+c c
\end{array}
$$

Now substituting Equations (14)-(15) and Equations (18)-(19) into Equations (12)-(13), the following are obtained

$$
\begin{aligned}
& \left(D_{0}^{2}+\omega_{1}^{2}\right) x_{2}= \\
& \left(-D_{1}^{2} A-2 i \omega_{1} \hat{c}_{1} A-2 i \omega_{1} D_{2} A+\Gamma_{1} A B \bar{B}+\Gamma_{2} A^{2} \bar{A}\right) \exp \left(i \omega_{1} T_{0}\right) \\
& +N S T+c c \\
& \left(D_{0}^{2}+\omega_{2}^{2}\right) y_{2}= \\
& \left(-D_{1}^{2} B-2 i \omega_{2} \hat{c}_{2} B-2 i \omega_{2} D_{2} B+\Gamma_{3} A \bar{A} B+\Gamma_{4} \bar{B} B^{2}\right) \exp \left(i \omega_{2} T_{0}\right) \\
& +\frac{1}{2} \hat{f}_{2} \bar{B} \exp \left(i\left(\Omega_{2}-\omega_{2}\right) T_{0}\right)+N S T+c c
\end{aligned}
$$

where

$$
\begin{gathered}
\Gamma_{1}=\left\{\frac{2 \hat{\beta}_{2} \hat{\alpha}_{3}}{\omega_{2}^{2}-\left(\omega_{1}+\omega_{2}\right)^{2}}+\frac{4 \hat{\alpha}_{2} \hat{\beta}_{2}}{\omega_{1}^{2}}-2 \hat{\eta}_{2}\right\}, \\
\Gamma_{2}=\left\{\frac{10 \hat{\alpha}_{2}^{2}}{3 \omega_{1}^{2}}-3 \hat{\gamma}_{2}\right\}, \\
\Gamma_{3}=\left\{\frac{\hat{\alpha}_{3}^{2}}{\left[\omega_{2}^{2}-\left(\omega_{1}+\omega_{2}\right)^{2}\right]}+\frac{2 \hat{\alpha}_{2} \hat{\alpha}_{3}}{\omega_{1}^{2}}-2 \hat{\eta}_{3}\right\}, \\
\Gamma_{4}=\left\{\frac{2 \hat{\beta}_{2} \hat{\alpha}_{3}}{\omega_{1}^{2}}-3 \hat{\gamma}_{3}\right\}
\end{gathered}
$$

and NST stands for non-secular terms. Eliminating the 
secular terms leads to the solvability conditions for the second-order expansion

$$
\begin{array}{r}
2 i \omega_{1} D_{2} A=-D_{1}^{2} A-2 i \omega_{1} \hat{c}_{1} A+\Gamma_{1} A B \bar{B}+\Gamma_{2} A^{2} \bar{A} \\
2 i \omega_{2} D_{2} B=-D_{1}^{2} B-2 i \omega_{2} \hat{c}_{2} B+\Gamma_{3} A \bar{A} B \\
+\Gamma_{4} \bar{B} B^{2}+\frac{1}{2} \hat{f}_{2} \bar{B} \exp \left(i \hat{\sigma}_{1} T_{1}\right)
\end{array}
$$

\section{Stability Analysis of Nonlinear Solutions}

From Equation (7), multiplying both sides be $2 i \omega_{1}$, $2 i \omega_{2}$ we get

$$
\begin{aligned}
2 i \omega_{1} \frac{\mathrm{d} A}{\mathrm{~d} t} & =\varepsilon 2 i \omega_{1} D_{1} A+\varepsilon^{2} 2 i \omega_{1} D_{2} A \\
2 i \omega_{2} \frac{\mathrm{d} B}{\mathrm{~d} t} & =\varepsilon 2 i \omega_{2} D_{1} B+\varepsilon^{2} 2 i \omega_{2} D_{2} B
\end{aligned}
$$

To analyze the solutions of Equations (16)-(17) and Equations (22)-(23), we express $A$ and $B$ in the polar form

$$
A\left(T_{1}, T_{2}\right)=(a / 2) e^{i \gamma_{1}}, B\left(T_{1}, T_{2}\right)=(b / 2) e^{i \gamma_{2}}
$$

where $a, b$ and $\gamma_{s}(s=1,2)$ are the steady state amplitudes and phases of the motion respectively. Substituting Equations (26), (16)-(17) and Equations (22)-(23) into Equations (24)-(25) and equating the real and imaginary parts we obtain the following equations describing the modulation of the amplitudes and phases:

$$
\begin{aligned}
\dot{a}= & -c_{1} a+\left\{\frac{\beta_{2}}{4 \omega_{1}}+\frac{\sigma_{2} \beta_{2}}{8 \omega_{1}^{2}}\right\} b^{2} \sin \theta_{2} \\
a \dot{\gamma}_{1}= & \left\{\frac{\beta_{2}}{4 \omega_{1}}+\frac{\sigma_{2} \beta_{2}}{8 \omega_{1}^{2}}\right\} b^{2} \cos \theta_{2} \\
& -\left\{\frac{\Gamma_{5}}{8 \omega_{1}}+\frac{\beta_{2} \alpha_{3}}{16 \omega_{1}^{2} \omega_{2}}\right\} a b^{2}-\frac{\Gamma_{6}}{8 \omega_{1}} a^{3} \\
\dot{b}=-c_{2} b+ & \left\{\frac{\sigma_{2} \alpha_{3}}{8 \omega_{2}^{2}}-\frac{\alpha_{3}}{4 \omega_{2}}\right\} a b \sin \theta_{2}+\frac{f_{2}}{4 \omega_{2}} b \sin \theta_{1} \\
b \dot{\gamma}_{2}= & \left\{\frac{\alpha_{3}}{4 \omega_{2}}-\frac{\sigma_{2} \alpha_{3}}{8 \omega_{2}^{2}}\right\} a b \cos \theta_{2}+\Gamma_{9} a^{2} b \\
& -\Gamma_{10} b^{3}-\frac{f_{2}}{4 \omega_{2}} b \cos \theta_{1}
\end{aligned}
$$

where

$$
\begin{aligned}
& \Gamma_{9}=\left\{\frac{\alpha_{3}^{2}}{32 \omega_{2}^{3}}-\frac{\Gamma_{7}}{8 \omega_{2}}\right\}, \Gamma_{10}=\left\{\frac{\beta_{2} \alpha_{3}}{32 \omega_{1} \omega_{2}^{2}}+\frac{\Gamma_{8}}{8 \omega_{2}}\right\} \\
& {\left[\Gamma_{5}, \Gamma_{6}, \Gamma_{7}, \Gamma_{8}\right]=\left[\varepsilon^{2} \Gamma_{1}, \varepsilon^{2} \Gamma_{2}, \varepsilon^{2} \Gamma_{3}, \varepsilon^{2} \Gamma_{4}\right]} \\
& \text { and } \theta_{1}=\hat{\sigma}_{1} T_{1}-2 \gamma_{2}, \theta_{2}=\hat{\sigma}_{2} T_{1}+\gamma_{1}-2 \gamma_{2}
\end{aligned}
$$

Form the system of Equations (27)-(30) to have stationary solutions, the following conditions must be satisfied:

$$
\dot{a}=\dot{b}=\dot{\theta}_{1}=\dot{\theta}_{2}=0
$$

It follows from Equation (31) that

$$
\dot{\gamma}_{2}=\frac{1}{2} \sigma_{1}, \dot{\gamma}_{1}=\sigma_{1}-\sigma_{2}
$$

Hence, the steady state solutions of Equations (27)-(30) are given by

$$
\begin{gathered}
c_{1} a-\left\{\frac{\beta_{2}}{4 \omega_{1}}+\frac{\sigma_{2} \beta_{2}}{8 \omega_{1}^{2}}\right\} b^{2} \sin \theta_{2}=0 \\
a\left(\sigma_{1}-\sigma_{2}\right)-\left\{\frac{\beta_{2}}{4 \omega_{1}}+\frac{\sigma_{2} \beta_{2}}{8 \omega_{1}^{2}}\right\} b^{2} \cos \theta_{2}+\frac{\Gamma_{6}}{8 \omega_{1}} a^{3} \\
+\left\{\frac{\Gamma_{5}}{8 \omega_{1}}+\frac{\beta_{2} \alpha_{3}}{16 \omega_{1}^{2} \omega_{2}}\right\} a b^{2}=0 \\
c_{2} b-\left\{\frac{\sigma_{2} \alpha_{3}}{8 \omega_{2}^{2}}-\frac{\alpha_{3}}{4 \omega_{2}}\right\} a b \sin \theta_{2}-\frac{f_{2}}{4 \omega_{2}} b \sin \theta_{1}=0 \\
\frac{1}{2} b \sigma_{1}+\left\{\frac{\sigma_{2} \alpha_{3}}{8 \omega_{2}^{2}}-\frac{\alpha_{3}}{4 \omega_{2}}\right\} a b \cos \theta_{2}-\Gamma_{9} a^{2} b \\
+\Gamma_{10} b^{3}+\frac{f_{2}}{4 \omega_{2}} b \cos \theta_{1}=0
\end{gathered}
$$

Solving the resulting algebraic equations for the fixed points of the practical case where $a \neq 0, b \neq 0$, that is non-planar motions, we obtain the following frequency response equations

$$
\begin{aligned}
& a^{2}\left(\sigma_{1}-\sigma_{2}\right)^{2}+c_{1}^{2} a^{2}+K_{1}^{2} a^{2} b^{4}+K_{2}^{2} a^{6}-K_{3} b^{4} \\
&+2\left(\sigma_{1}-\sigma_{2}\right) K_{1} a^{2} b^{2}+2\left(\sigma_{1}-\sigma_{2}\right) K_{2} a^{6} \\
&+2 K_{1} K_{2} a^{4} b^{2}=0 \\
& \frac{1}{4} b^{2} \sigma_{1}^{2}+c_{2}^{2} b^{2}+\Gamma_{9}^{2} a^{4} b^{2}+\Gamma_{10}^{2} b^{6}-\Gamma_{9} \sigma_{1} b^{2} a^{2} \\
&+\Gamma_{10} \sigma_{1} b^{4}-2 \Gamma_{9} \Gamma_{10} a^{2} b^{4}-K_{4} a^{2} b^{2}-\frac{f_{2}^{2}}{16 \omega_{2}^{2}} b^{2} \\
&-\frac{K_{4} f_{2}}{2 \omega_{2}} a b^{2} \cos \left(\theta_{1}-\theta_{2}\right)=0
\end{aligned}
$$

where

$$
\begin{aligned}
& K_{1}=\left\{\frac{\Gamma_{5}}{8 \omega_{1}}+\frac{\beta_{2} \alpha_{3}}{16 \omega_{1}^{2} \omega_{2}}\right\}, K_{2}=\frac{\Gamma_{6}}{8 \omega_{1}}, K_{3}=\left\{\frac{\beta_{2}}{4 \omega_{1}}+\frac{\sigma_{2} \beta_{2}}{8 \omega_{1}^{2}}\right\} \\
& \text { and } K_{4}=\left\{\frac{\sigma_{2} \alpha_{3}}{8 \omega_{2}^{2}}-\frac{\alpha_{3}}{4 \omega_{2}}\right\} .
\end{aligned}
$$

The stability of the obtained fixed points for the simul- 
taneous primary, principal parametric and 2:1 internal resonance case is determined and studied as follows: one lets

$$
\begin{aligned}
& a=a_{10}+a_{11}, b=b_{10}+b_{11} \\
& \text { and } \theta_{s}=\theta_{s 0}+\theta_{s 1}
\end{aligned}
$$

where $a_{10}, b_{10}$ and $\theta_{s 0}$ are the solutions of Equations (34)-(37) and $a_{11}, b_{11}, \theta_{s 1}$ are perturbations which are assumed to be small compared to $a_{10}, b_{10}$ and $\theta_{s 0}$. Substituting Equation (40) into Equations (27)-(30), using Equations (34)-(37) and keeping only the linear terms in $a_{11}, b_{11}, \theta_{s 1}$ we obtain:

$$
\begin{aligned}
& \dot{a}_{11}=-c 1 a 11+\left\{K_{3} b_{10}^{2} \cos \theta_{20}\right\} \theta_{21} \\
& +\left\{2 K_{3} b_{10} \sin \theta_{20}\right\} b_{11} \\
& \dot{\theta}_{21} \\
& =\left\{\frac{\left(\sigma_{2}-\sigma_{1}\right)}{a_{10}}-\frac{K_{1} b_{10}^{2}}{a_{10}}-\frac{3 \Gamma_{6} a_{10}}{8 \omega_{1}}+K_{4} \cos \theta_{20}-2 \Gamma_{9} a_{10}\right\} a_{11} \\
& +\left\{-K_{4} a_{10} \sin \theta_{20}-\frac{K_{3} b_{10}^{2}}{a_{10}} \sin \theta_{20}\right\} \theta_{21} \\
& +\left\{\frac{\sigma_{1}}{2 b_{10}}+\frac{2 K_{3} b_{10}}{a_{10}} \cos \theta_{20}-2 K_{1} b_{10}+\frac{K_{4} a_{10}}{b_{10}} \cos \theta_{20}\right. \\
& \left.-\frac{\Gamma_{6} a_{10}^{2}}{b_{10}}+3 \Gamma_{10} b_{10}+\frac{f_{2}}{4 \omega_{2}} \cos \theta_{10}\right\} b_{11} \\
& -\left\{\frac{f_{2}}{2 \omega_{2}} \sin \theta_{10}\right\} \theta_{11} \\
& \dot{b}_{11}=\left\{K_{4} b_{10} \sin \theta_{20}\right\} a_{11} \\
& +\left\{-c_{2}+K_{4} a_{10} \sin \theta_{20}+\frac{f_{2}}{4 \omega_{2}} \sin \theta_{10}\right\} b_{11} \\
& +\left\{K_{4} a_{10} b_{10} \cos \theta_{20}\right\} \theta_{21}+\left\{\frac{f_{2}}{4 \omega_{2}} b_{10} \cos \theta_{10}\right\} \theta_{11} \\
& \dot{\theta}_{11}=\left\{K_{4} \cos \theta_{20}-2 \Gamma_{9} a_{10}\right\} a_{11}-\left\{K_{4} a_{10} \sin \theta_{20}\right\} \theta_{21} \\
& +\left\{\frac{\sigma_{1}}{2 b_{10}}+\frac{K_{4} a_{10}}{b_{10}} \cos \theta_{20}-\frac{\Gamma_{9} a_{10}^{2}}{b_{10}}+3 \Gamma_{10} b_{10}+\frac{f_{2}}{4 \omega_{2}} \cos \theta_{10}\right\} b_{11} \\
& -\left\{\frac{f_{2}}{2 \omega_{2}} \sin \theta_{10}\right\} \theta_{11}
\end{aligned}
$$

The system of Equations (41)-(44) are first order autonomous ordinary differential equations and the stability of a particular fixed point with respect to an infinitesimal disturbance proportional to $\exp (\lambda t)$ is determined by eigenvalues of the Jacobian matrix of the right hand sides of Equations (41)-(44). The zeros of the char- acteristic equation are given by

$$
\lambda^{4}+L_{1} \lambda^{3}+L_{2} \lambda^{2}+L_{3} \lambda+L_{4}=0
$$

where, $L_{1}, L_{2}, L_{3}$ and $L_{4}$ are functions of the parameters $\left(a, b, \omega_{1}, \omega_{2}, c_{1}, c_{2}, \alpha_{2}, \alpha_{3}, \beta_{2}, \eta_{2}, \eta_{3}, \gamma_{2}, \gamma_{3}, f_{2}, \theta_{1}, \sigma_{1}, \sigma_{2}\right)$. According to the Routh-Hurwitz criterion the necessary and sufficient conditions for all the roots of Equation (45) to possess negative real parts are:

$$
L_{1}>0, L_{1} L_{2}-L_{3}>0, L_{3}\left(L_{1} L_{2}-L_{3}\right)-L_{1}^{2} L_{4}>0, L_{4}>0
$$

The system is stable if the eigenvalues have negative real parts, otherwise is unstable. In the frequency response curves, solid/dotted lines denote stable/ unstable periodic responses, respectively.

\section{Results and Discussion}

The response of the two-degree-of-freedom nonlinear system under both parametric and external excitations is studied. The solution of this system is determined up to and including the second order approximation by applying the multiple time scale perturbation. The steady state solution and its stability are determined and representative numerical results are included. The stability zone and effects of the different parameters are discussed using frequency response curve. The stability of the numerical solution is studied also using the phase-plane method. Some of the resulting resonance cases are confirmed applying well-known numerical techniques. The effects of the some different parameters on the vibrating system behavior are investigated and discussed.

\subsection{Numerical Solution}

Figure 2 shows that the response of the inclined cable for the non-resonant at the practical values of the parameters $c_{1}=0.0002, c_{2}=0.03, \alpha_{2}=0.2, \beta_{2}=0.5, \gamma_{2}=$ $0.3, \eta_{2}=0.5, \alpha_{3}=0.03, \eta_{3}=0.05, \gamma_{3}=0.04, f_{1}=2, f_{2}=$ $0.01, \Omega_{1}=2.75, \Omega_{2}=3.2, \omega_{1}=1.2, \omega_{2}=1.5$. It can be seen from this figure that the steady state amplitude is about 0.005 with dynamic chaotic behavior for the inplane mode and about 0.18 with multi-limit cycle for the out-of-plane mode. The amplitudes decreasing with increasing time and tend to steady state motion and have stable solution. The worst resonance case is also confirmed numerically as shown in Figure 3. From this figure, it can be notice that the maximum steady state amplitude of the in-plane mode is about 130 times that of basic case with multi-limit cycle, while the maximum amplitude of out-of-plane mode is about 4 times of the basic case with chaotic motion.

Effects of external and parametric excitation forces $f_{1}$ and $f_{2}$. 

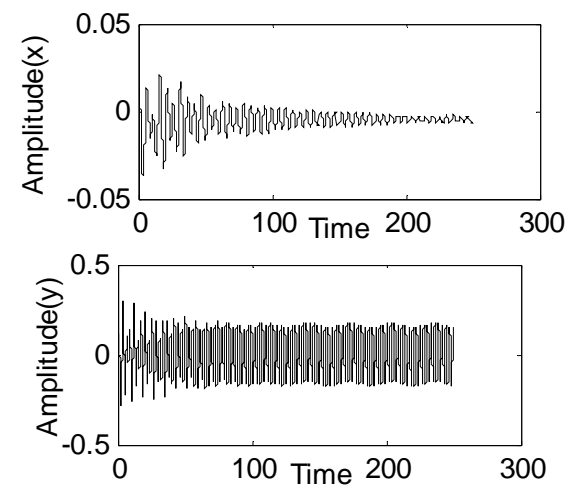

Figure 2. Non-resonance system behavior (basic case) $\Omega_{1} \neq$ $\omega_{1} \neq \omega_{2}$.
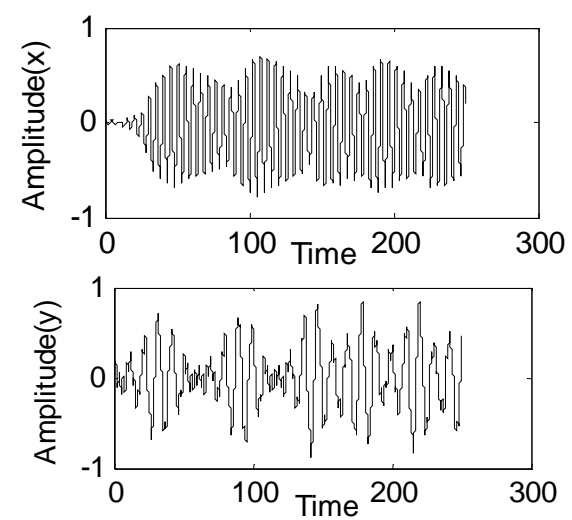

Figure 3. Simultaneous principal parametric resonance in the presence of 2:1 internal resonance $\left(\Omega_{2} \cong 2 \omega_{2}\right.$ and $\left.\omega_{1} \cong 2 \omega_{2}\right)$.

For increasing the amplitude of the external or parametric excitation forces $f_{1}$ or $f_{2}$, we observe that the modes of vibration have increasing magnitudes and there exist chaotic dynamic motion as shown in Figures $\mathbf{4}$ and $\mathbf{5}$.

\subsection{Frequency Response Curves}

The frequency response Equations (38)-(39) are nonlinear algebraic equations in the amplitudes of the system $a$ (in-plane mode) and $b$ (out-of-plane mode). The stability of a fixed point solution is studied by examination of the eigenvalues of Equation (45). The numerical results of Equations (38) and (39) are plotted in Figures 6-8.

Figure 6, show the frequency response curves of the two modes of inclined cable against detuning parameter $\sigma_{1}$. From the geometry of the figures we observe that the amplitudes have two branches and these branches are bent to the right, the bending leads to multi-valued solutions and hence the effective nonlinearity is hardening type. In Figure 6(a), there are two branches of nontrivial solution such that the left branch stable and the right branch lose stability as $\sigma_{1} \leq 0.4$. Figure 6(b), show that the steady state amplitudes are increasing for increasing
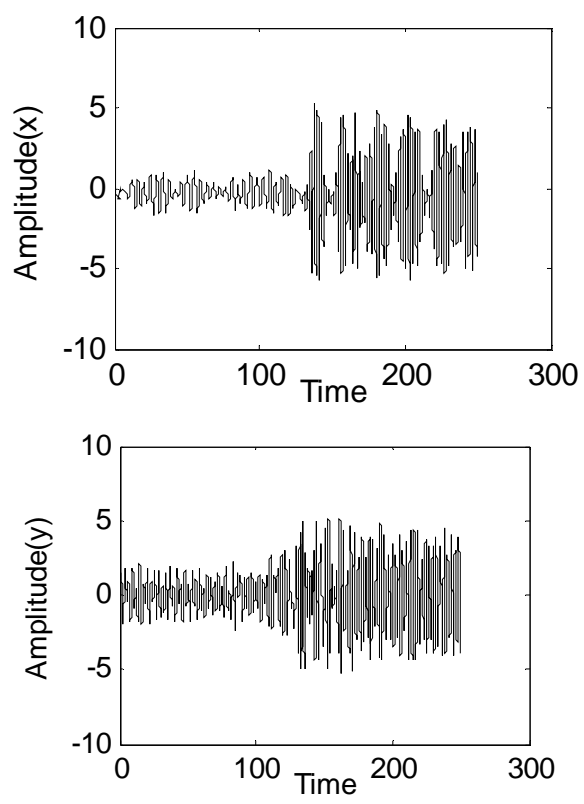

Figure 4. Effects of increasing value of external excitation force $f_{1}=5$.
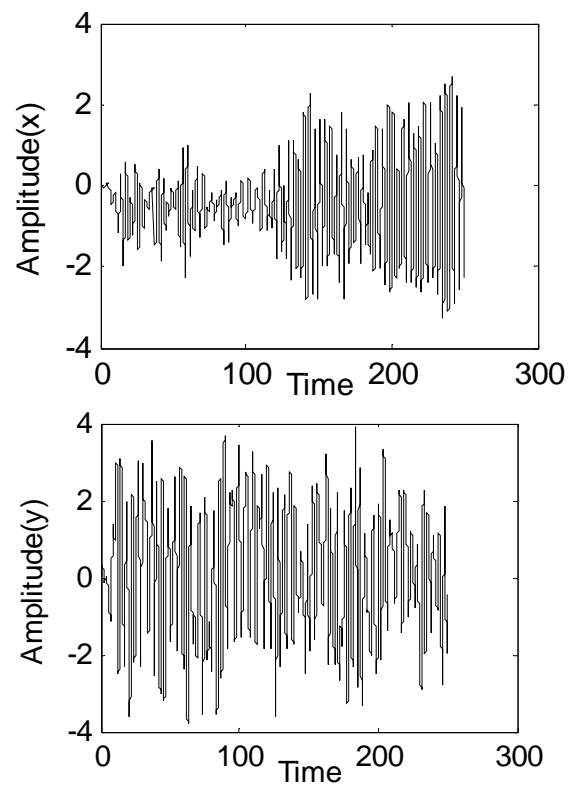

Figure 5. Effects of increasing value of parametric excitation force $f_{2}=3$.

parametric excitation force $f_{2}$. The region of instability for two modes is increasing for increasing $f_{2}$. For in creasing nonlinear parameter $\beta_{2}$ (i.e. $\beta_{2}=1$ ) as shown in Figure 6(c), we show that the regions of definition are decreasing and the two branches of the steady state amplitude curve are contracted and give one continuous curve which is stable and response amplitude of the inplane mode is increased. Figure 6(d) show that the response amplitudes of the inclined cable are increasing for 

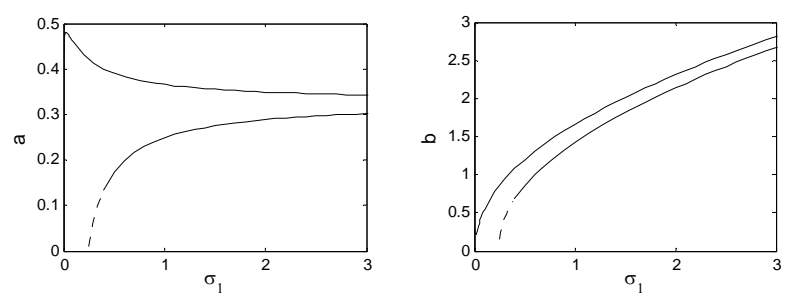

(a)
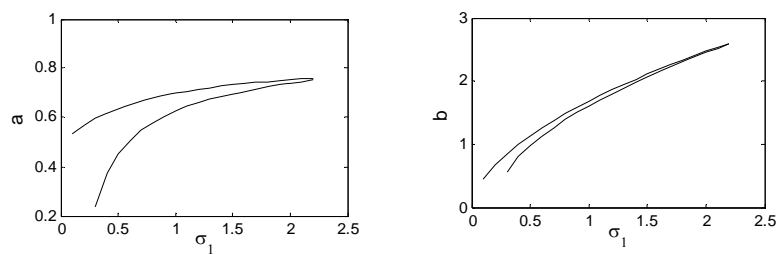

(c)

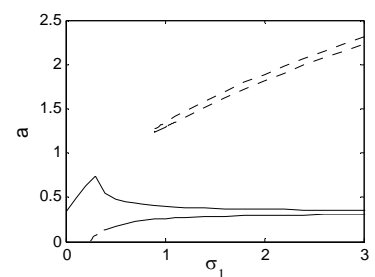

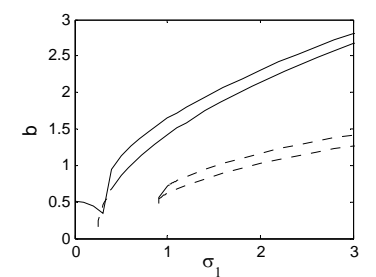

(e)
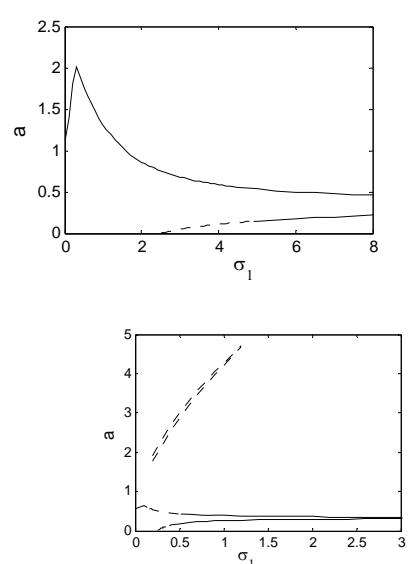

(b)
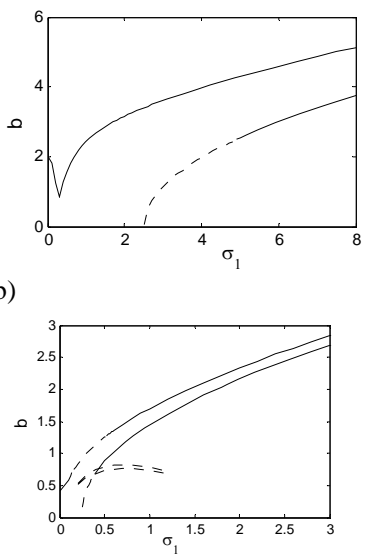

(d)
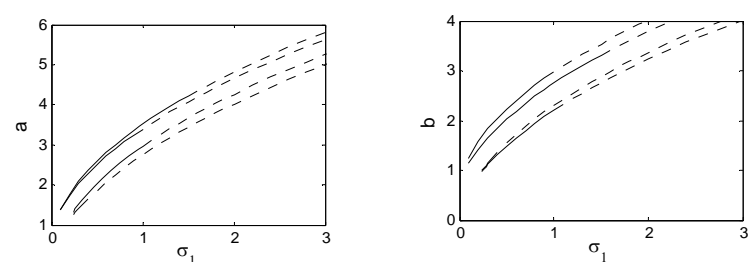

(f)

Figure 6. (a): Frequency response curves for amplitudes against $\sigma_{1}$; (b): Frequency response curve for increasing parametric excitation force $f_{2}=3.0 ;(\mathrm{c})$ : Frequency response curve for increasing nonlinear parameter $\beta_{2}=1.0$; (d): Frequency response curve for decreasing nonlinear parameter $\eta_{3}=0.1$; (e): Frequency response curve for increasing nonlinear parameter $\gamma_{2}=1.8$; (f): Frequency response curve for negative value of nonlinear parameter $\gamma_{3}=-\mathbf{0 . 4}$.

decreasing nonlinear parameter $\eta_{3}$ and the regions of multi-valued and instability of two modes are increasing. The regions of instability solutions are increasing for increasing nonlinear parameter $\gamma_{2}$ as shown in Figure 6(e). Figure 6(f) shows that for negative value of nonlinear parameter $\gamma_{3}$ the response amplitudes are increasing and the stability solution are decreasing with increasing region of multi-valued.

Figure 7, represent the variation of the amplitudes of the inclined cable against the detuning parameter $\sigma_{2}$. In Figure 7(a), we see that each mode of the inclined cable has one continuous curve and single valued solution and it is symmetric about the origin and it is noticed that the in-plane mode reaches maximum value at $\sigma_{2}=0$ and the out-of-plane mode reaches minimum value at the same value of $\sigma_{2}$. Also, it intersects in two points and these modes have stable and unstable solutions. From Figure 7(b), we observe that for increasing parametric excitation force $f_{2}$ the symmetric branch moves up with increased magnitudes and the region of stability is increased. For increasing nonlinear parameter $\gamma_{3}$, we note that the amplitudes of the two modes of the inclined cable have decreasing magnitudes and increasing stable solutions, as shown in Figure 7(c). The steady state amplitudes of the two modes are increasing for decreasing nonlinear parameter $\eta_{3}$ as shown in Figure 7(d). Also, the region of stability solutions is increased. From Figure 7(e) we observe that the steady state amplitudes $a$ and $b$ of the two modes are increasing for decreasing value of nonlinear parameters $\alpha_{3}$ respectively with increasing stable solutions. The stability solution is decreasing as the nonlinear parameter $\alpha_{2}$ is increase and the curves are shifted to the right and has hardening phenomena and there exists jump phenomena, as shown in Figure 7(f).

Figure 8 represent force-response curves for the nonlinear solution of the case of simultaneous principal parametric resonance in the presence of 2:1 internal resonances. In this figure the amplitudes of the inclined cable are plotted as a function of the parametric excitation force $f_{2}$. Figure 8 shows that the response amplitudes of the inclined cable have a continuous curve and the curve has stable and unstable solutions.

\section{Comparison with Published Work}

In comparison with the previous work [8], we have the global bifurcation of this inclined cable leading to primary resonances and 1:1 internal resonance is investigated. A new global perturbation technique is employed to analyze Shilnikov type homoclinic orbits and chaotic dynamics in the inclined cable. Kamel and Hamed [9], 


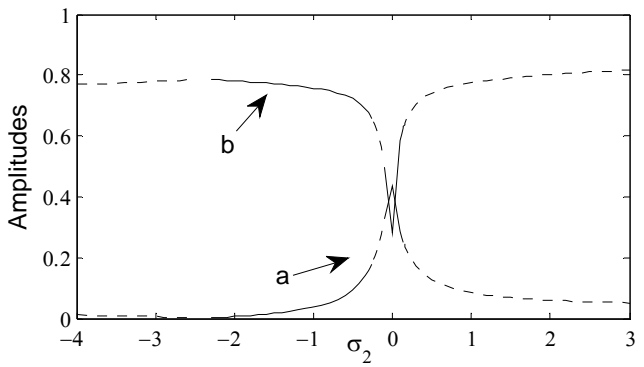

(a)

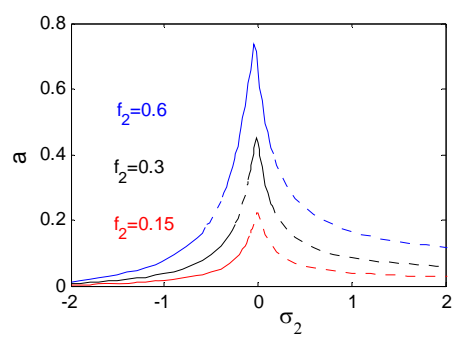

(b)
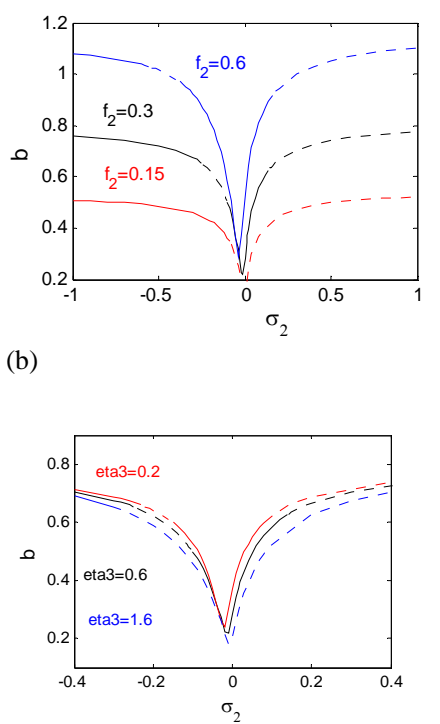

(d)
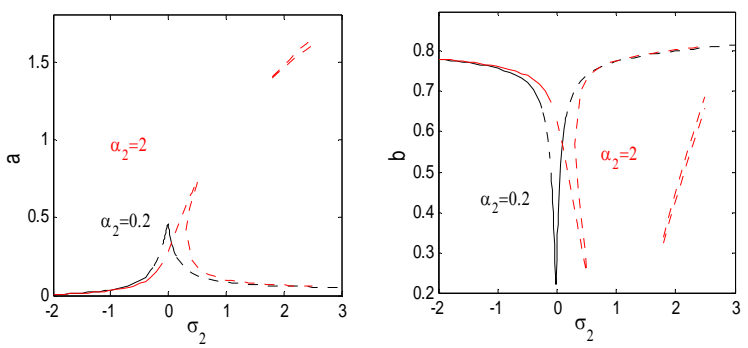

(f)

Figure 7. (a): Frequency response curves for simultaneous principal parametric resonance in the presence of 2:1 internal resonance $\Omega_{2} \cong 2 \omega_{2}$ and $\omega_{1} \cong 2 \omega_{2}$; (b): Frequency response curve for parametric excitation force $f_{2}$; (c): Frequency response curve for nonlinear parameter $\gamma_{3}$; (d): Frequency response curve for nonlinear parameter $\eta_{3}$; (e): Frequency response curve for nonlinear parameter $\alpha_{3}$; (f): Frequency response curve for nonlinear parameter $\alpha_{2}$.
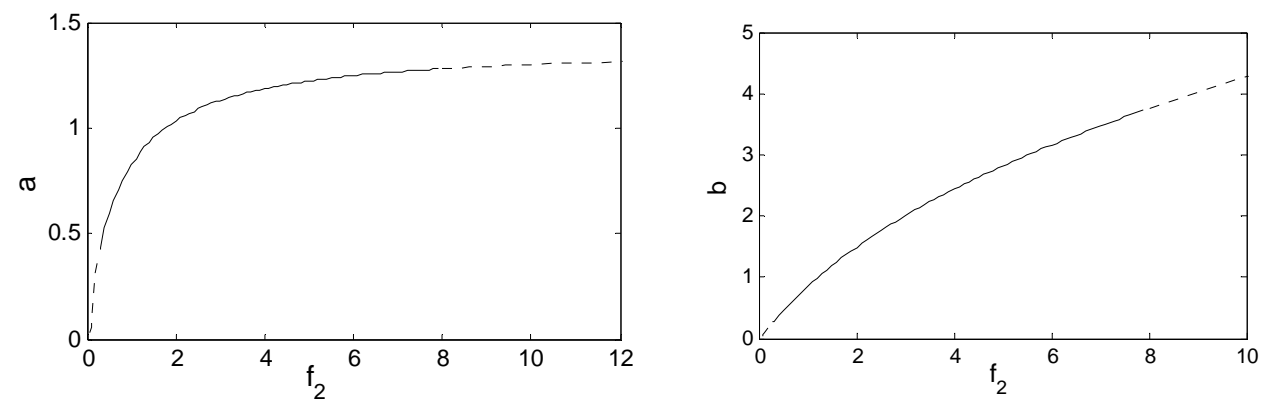

Figure 8. Force response curves for $\left(\Omega_{2} \cong 2 \omega_{2}, \omega_{1} \cong 2 \omega_{2}\right)$.

studied the nonlinear behavior of an inclined cable subjected to harmonic excitation near the simultaneous primary and 1:1 internal resonance by using multiple scale method.

In this paper, periodic and chaotic response of a discretization two-degree-of-freedom model of a suspended inclined cable, containing a 2:1 internal resonance, sub- ject to harmonic external and parametric excitation are obtained. The stable/unstable periodic solutions are determined using the method of multiple scale and are presented through frequency response plots. Chaotic responses are determined by numerical integration of the governing ordinary differential equations of motion. Variation of the parameters $\alpha_{2}, \alpha_{3}, \beta_{2}, \gamma_{2}, \eta_{2}, \gamma_{3}, \eta_{3}, f_{2}$ leads 
to multi-valued amplitudes and hence to jump phenomena.

\section{Conclusions}

Cables are very efficient structural members and hence have been widely used in many long-span structures, including suspension, roofs and guyed towers. The nonlinear dynamic response of the nonlinear system subjected to external and parametric excitations is investigated. The method of multiple scales is applied to obtain the solution of the considered system up to second order approximation. The numerical solutions and chaotic response of this nonlinear system are investigated. The stability of the proposed analytic nonlinear solution is studied at worst resonance case which is the simultaneous principal parametric resonance in the presence of 2:1 internal resonances. The modulation equations of the amplitudes and phases are obtained and steady state solutions are determined. The effects of some nonlinear parameters on the steady state response of the vibrating cable leading to multi-valued solutions. From the analysis the following may be concluded.

1) For the resonance case $\Omega_{2} \cong 2 \omega_{2}, \omega_{1} \cong 2 \omega_{2}$ we note that the steady state amplitude is increased to about $130 \%$ compared to basic case with multi-limit cycle, and it is better to avoid this resonance case as working conditions for the system.

2) The steady state amplitude of the system are increasing for increasing external or parametric excitation force, and for large values of the system become unstable.

3) Variation of $\alpha_{2}, \alpha_{3}, \beta_{2}, \gamma_{2}, \eta_{2}, \gamma_{3}, \eta_{3}, f_{2}$ leads to multi-valued amplitudes and hence jump phenomena.

4) For increasing parametric excitation force $f_{2}$ or negative value of the nonlinear parameter $\gamma_{3}$ we observe that the steady state amplitudes of the two modes are increasing with increasing instability solutions.

5) Increasing of the nonlinear parameters $\eta_{3}$ or $\gamma_{3}$ can reduce the amplitude of the system and obtain the effect of reduction of the amplitude.

6) Variation of the parameter $\alpha_{2}$ leads to multi-valued amplitudes and hence to jump phenomena.

7) For increasing parametric excitation force $f_{2}$ or decreasing nonlinear parameter $\alpha_{3}$ we show that the steady state amplitudes of the two modes are increasing.

For increasing nonlinear parameter $\eta_{3}$ we note that the steady state amplitudes of the two modes are decreasing with decrease of the stability solutions.

\section{References}

[1] H. N. Arafat and A. H. Nayfeh, "Non-Linear Responses of Suspended Cables to Primary Resonance Excitations,”
Journal of Sound and Vibration, Vol. 266, No. 2, 2003, pp. 325-354. doi:10.1016/S0022-460X(02)01393-7

[2] G. Rega, "Non-Linear Vibrations of Suspended Cables; Part I: Modeling and Analysis," Journal of Applied Mechanics Review, Vol. 57, No. 6, 2004, pp. 443-478. doi:10.1115/1.1777224

[3] G. Rega, "Non-Linear Vibrations of Suspended Cables; Part II: Deterministic Phenomena,” Journal of Applied Mechanics Review, Vol. 57, No. 6, 2004, pp. 479-514. doi:10.1115/1.1777225

[4] S. R. Nielsen and P. H. Kirkegaard, "Super and Combinatorial Harmonic Response of Flexible Inclined Cables with Small Sag," Journal of Sound and Vibration, Vol. 251, No. 1, 2002, pp. 79-102. doi:10.1006/jsvi.2001.3979

[5] G. Zheng, J. M. Ko and Y. O. Ni, "Super-Harmonic and Internal Resonances of a Suspended Cable with Nearly Commensurable Natural Frequencies," Nonlinear Dynamics, Vol. 30, No. 1, 2002, pp. 55-70. doi:10.1023/A:1020395922392

[6] W. Zhang and Y. Tang, "Global Dynamics of the Cable under Combined Parametrical and External Excitations," International Journal of Non-Linear Mechanics, Vol. 37, No. 3, 2002, pp. 505-526. doi:10.1016/S0020-7462(01)00026-9

[7] A. H. Nayfeh, H. Arafat, C. M. Chin and W. Lacarbonara, "Multimode Interactions in Suspended Cables," Journal of Vibration and Control, Vol. 8, No. 3, 2002, pp. 337387. doi:10.1177/107754602023687

[8] H. Chen and Q. Xu, "Bifurcation and Chaos of an Inclined Cable,” Nonlinear Dynamics, Vol. 57, No. 2-3, 2009, pp. 37-55. doi:10.1007/s11071-008-9418-3

[9] M. M. Kamel and Y. S. Hamed, "Non-Linear Analysis of an Inclined Cable under Harmonic Excitation," Acta Mechanica, Vol. 214, No. 3-4, 2010, pp. 315-325. doi:10.1007/s00707-010-0293-X

[10] A. Abe, "Validity and Accuracy of Solutions for Nonlinear Vibration Analyses of Suspended Cables with Oneto-One Internal Resonance," Nonlinear Analysis: Real World Applications, Vol. 11, No. 4, 2010, pp. 2594-2602. doi:10.1016/j.nonrwa.2009.09.006

[11] N. Srinil, G. Rega and S. Chucheepsakul, "Two-yo-One Resonant Multi-Modal Dynamics of Horizontal/Inclined Cables. Part I: Theoretical Formulation and Model Validation,” Nonlinear Dynamics, Vol. 48, No. 3, 2007, pp. 231-252. doi:10.1007/s11071-006-9086-0

[12] N. Srinil and G. Rega, "Two-To-One Resonant MultiModal Dynamics of Horizontal/Inclined Cables. Part II: Internal Resonance Activation Reduced-Order Models and Nonlinear Normal Modes,” Nonlinear Dynamics, Vol. 48, No. 3, 2007, pp. 253-274. doi:10.1007/s11071-006-9087-z

[13] R. Alaggio and G. Rega, "Characterizing Bifurcations and Classes of Motion in the Transition to Chaos through 3D-Tori of a Continuous Experimental System in Solid Mechanics," Physica D, Vol. 137, No. 1, 2000, pp. 70-93. doi:10.1016/S0167-2789(99)00169-4

[14] G. Rega and R. Alaggio, "Spatio-Temporal Dimensional- 
ity in the Overall Complex Dynamics of an Experimental Cable/Mass System," International Journal of Solids and Structures, Vol. 38, No. 10-13, 2001, pp. 2049-2068. doi:10.1016/S0020-7683(00)00152-9

[15] A. Gonzalez-Buelga, S. A. Neild, D. J. Wagg and J. H. G. Macdonald, "Modal Stability of Inclined Cables Subjected to Vertical Support Excitation,” Journal of Sound and Vibration, Vol. 318, No. 3, 2008, pp. 565-579. doi:10.1016/j.jsv.2008.04.031

[16] N. C. Perkins, "Modal Interactions in the Non-Linear Response of Inclined Cables under Parametric/External Excitation," International Journal of Non-linear Mechanics, Vol. 27, No. 2, 1992, pp. 233-250. doi:10.1016/0020-7462(92)90083-J

[17] C. L. Lee and N. C. Perkins, "Nonlinear Oscillations of Suspended Cables Containing a Two-to-One Internal Resonance,” Nonlinear Dynamics, Vol. 3, 1992, pp. 465490.

[18] C. L. Lee and N. C. Perkins, "Three-Dimensional Oscillations of Suspended Cables Involving Simultaneous Internal Resonance," Proceedings of ASME Winter Annual Meeting AMD-14, 1992, pp. 59-67.

[19] M. Eissa and M. Sayed, “A Comparison between Passive and Active Control of Non-Linear Simple Pendulum Part-I," Mathematical and Computational Applications, Vol. 11, No. 2, 2006, pp. 137-149.

[20] M. Eissa and M. Sayed, “A Comparison between Passive and Active Control of Non-Linear Simple Pendulum Part-II," Mathematical and Computational Applications, Vol. 11, No. 2, 2006, pp. 151-162.

[21] M. Eissa and M. Sayed, "Vibration Reduction of a Three DOF Non-Linear Spring Pendulum,” Communication in
Nonlinear Science and Numerical Simulation, Vol. 13, No. 2, 2008, pp. 465-488.

doi:10.1016/j.cnsns.2006.04.001

[22] M. Sayed, "Improving the Mathematical Solutions of Nonlinear Differential Equations Using Different Control Methods,” Ph.D. Thesis, Menofia University, Egypt, November 2006.

[23] M. Sayed and Y. S. Hamed, "Stability and Response of a Nonlinear Coupled Pitch-Roll Ship Model under Parametric and Harmonic Excitations," Nonlinear Dynamics, Vol. 64, No. 3, 2011, pp. 207-220. doi:10.1007/s11071-010-9841-0

[24] M. Sayed and M. Kamel, "Stability Study and Control of Helicopter Blade Flapping Vibrations," Applied Mathematical Modelling, Vol. 35, No. 6, 2011, pp. 2820-2837. doi:10.1016/j.apm.2010.12.002

[25] M. Sayed and M. Kamel, “1:2 and 1:3 Internal Resonance Active Absorber for Non-Linear Vibrating System," $A p$ plied Mathematical Modelling, Vol. 36, No. 1, 2012, pp. 310-332. doi:10.1016/j.apm.2011.05.057

[26] Y. A. Amer and M. Sayed, "Stability at Principal Resonance of Multi-Parametrically and Externally Excited Mechanical System," Advances in Theoretical and Applied Mechanics, Vol. 4, No. 1, 2011, pp. 1-14.

[27] M. Sayed, Y. S. Hamed and Y. A. Amer, "Vibration Reduction and Stability of Non-Linear System Subjected to External and Parametric Excitation Forces under a Nonlinear Absorber," International Journal of Contemporary Mathematical Sciences, Vol. 6, No. 22, 2011, pp. 1051 1070.

[28] A. H. Nayfeh, "Non-Linear Interactions," Wiley/InterScience, New York, 2000. 